\title{
High energy Coulomb-scattered electrons for relativistic particle beam diagnostics
}

\author{
P. Thieberger," Z. Altinbas, C. Carlson, C. Chasman, M. Costanzo, C. Degen, K. A. Drees, \\ W. Fischer, D. Gassner, X. Gu, K. Hamdi, J. Hock, A. Marusic, T. Miller, M. Minty, \\ C. Montag, Y. Luo, and A. I. Pikin \\ Brookhaven National Laboratory, Upton, New York 11973, USA
}

S. M. White

European Synchrotron Radiation Facility, BP 220, 38043 Grenoble Cedex, France

(Received 25 September 2015; published 22 April 2016)

\begin{abstract}
A new system used for monitoring energetic Coulomb-scattered electrons as the main diagnostic for accurately aligning the electron and ion beams in the new Relativistic Heavy Ion Collider (RHIC) electron lenses is described in detail. The theory of electron scattering from relativistic ions is developed and applied to the design and implementation of the system used to achieve and maintain the alignment. Commissioning with gold and ${ }^{3} \mathrm{He}$ beams is then described as well as the successful utilization of the new system during the 2015 RHIC polarized proton run. Systematic errors of the new method are then estimated. Finally, some possible future applications of Coulomb-scattered electrons for beam diagnostics are briefly discussed.
\end{abstract}

DOI: 10.1103/PhysRevAccelBeams.19.041002

\section{INTRODUCTION}

Instrumentation providing accurate information on particle beam properties and behavior in accelerators is essential for their operation. The challenge of performing reliable and often delicate measurements in the harsh particle accelerator environment provides strong incentives for exploring new approaches. We describe a new type of beam diagnostic device for high energy particle accelerators based on the Coulomb scattering of electrons by the beam particles. Measuring the deflection of low energy electron beams by the macroscopic fields generated by the high energy particle beam to be characterized, the so-called electron wire, was proposed in the late 1980s and early 1990s [1-4] and later implemented in several systems, including the use of electron ribbons instead of the pencil beams [5-8]. The system we describe here is a new noninvasive beam diagnostic tool also based on the Coulomb interaction of low energy electrons with relativistic particle beams, but in this case the interaction is due to small impact parameter collisions of a small fraction of the electrons with individual beam particles leading to large momentum transfers. This mechanism is the so-called Rutherford scattering, named after Ernest Rutherford

\footnotetext{
* Corresponding author. PT@BNL.GOV
}

Published by the American Physical Society under the terms of the Creative Commons Attribution 3.0 License. Further distribution of this work must maintain attribution to the author $(s)$ and the published article's title, journal citation, and DOI. who, in 1911 [9], discovered the atomic nucleus by studying the scattering of alpha and beta particles from stationary targets. Our targets, the ion beam particles, far from being stationary, are moving at relativistic velocities. The theory describing the interaction is the same in the frame of reference comoving with the particle beam. Using this theory, we can predict the energies and angular distribution of the scattered electrons by coordinate transformation to the laboratory frame. In the laboratory frame, some of these electrons acquire energies up to several $\mathrm{MeV}$, making them easy to detect even after traversing thin vacuum windows, thus allowing the use of simple scintillation detectors in air.

Based on these ideas, we developed a noninvasive diagnostic tool called electron beam backscattering detector (eBSD) [10] to accurately align the electron and proton beams in the new Brookhaven National Laboratory (BNL) electron lenses for the partial compensation of the head-on beam-beam effects that limit the luminosity [11].

In the following sections we review the theory of electrons scattered form relativistic ions, we then describe the principle of applying this phenomenon to the alignment of electron and ion beams in the RHIC electron lenses and we describe the implementation of the backscattered electron detectors. We then report on the commissioning of the systems with gold and helium beams and, finally, we describe in detail the successful utilization of this new diagnostic instrument during the 2015 RHIC polarized proton-proton operations run (henceforth: "run"). Based on the experience and data from these runs, we then provide an analysis and estimates of systematic errors of this alignment method. 
During the first commissioning run of these systems $[10,12]$ it was discovered that energetic scattered electrons are also generated by the interaction of the relativistic particles with the electrons of residual gas atoms. We mention here the possible use of these electrons in other noninvasive beam diagnostic instruments not requiring a low energy electron beam, and we suggest the possibility of using instruments similar to the eBSD for the alignment of other configurations involving ion and electron beams such as hollow beams for collimation or for halo monitoring, and long range beam-beam compensators [13].

Finally, we suggest that detecting the electrons scattered from an "electron wire" may be an alternative, and more intensity-independent way, to obtain profiles as compared to the present measurements of small deflections caused by the macroscopic fields generated by the beam. Time resolved measurements of this type, in addition to providing transverse beam profiles, could also provide longitudinal bunch profiles and diagnostics for "head-tail" perturbations.

\section{THEORY OF ELECTRONS SCATTERED FROM MOVING TARGETS}

To first order in the fine structure constant, the Coulomb scattering of relativistic electrons by nuclei is described by the Mott formula which in the rest frame of the nucleus is written [14]

$$
\begin{aligned}
\frac{d \sigma}{d \Omega}= & \frac{Z^{2}}{4}\left(\frac{e^{2}}{E}\right)^{2} \frac{1}{\sin ^{4}(\theta / 2)} \times\left[1-\left(\frac{p c}{E}\right)^{2} \sin ^{2} \frac{\theta}{2}\right] \\
& \times\left[1+\frac{2 E \sin ^{2}(\theta / 2)}{M_{A} c^{2}}\right]^{-1},
\end{aligned}
$$

where $\Omega$ is the solid angle, $\sigma$ the cross section, $Z$ the atomic number, $\mathrm{M}_{A}$ the mass of the nucleus, $e$ the elementary

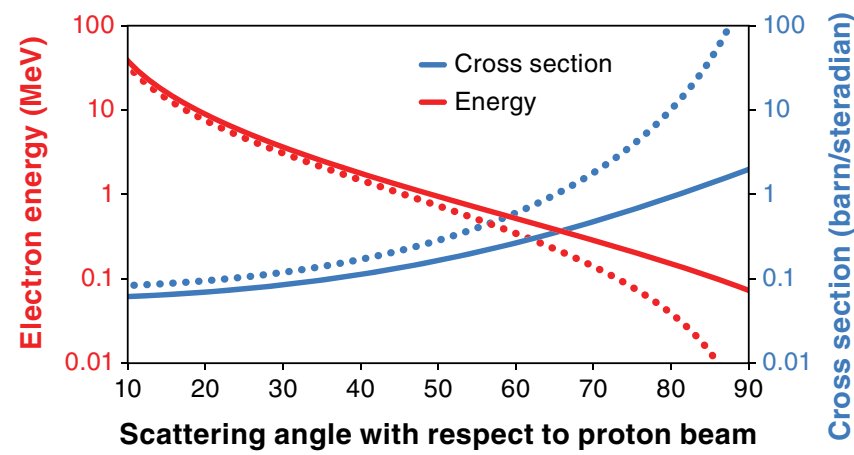

FIG. 1. The solid lines show calculated energies and approximate scattering cross sections for $5 \mathrm{keV}$ electrons backscattered by $250 \mathrm{GeV}$ protons. The dotted lines correspond to the same quantities, but for $10 \mathrm{eV}$ electrons as a qualitative indication of energetic electrons generated by the interaction of the beam with the residual gas and/or with low energy electrons captured in the potential well of the beam.

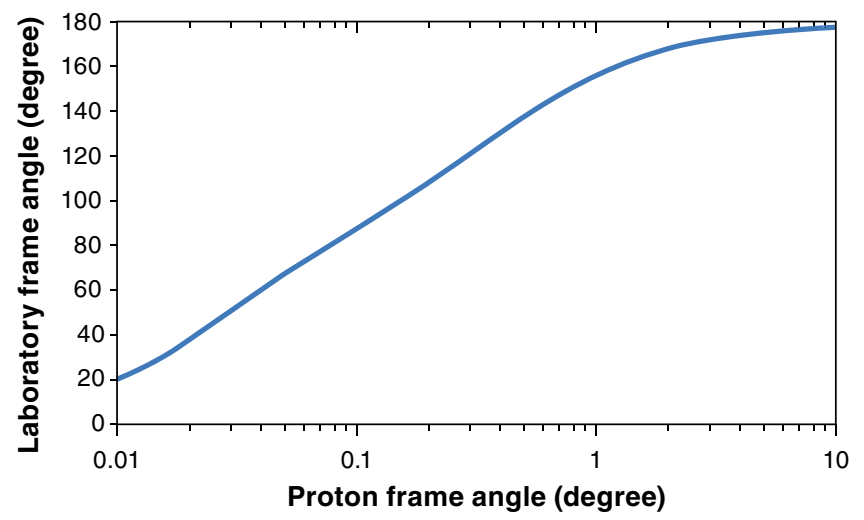

FIG. 2. Angles with respect to the initial electron beam direction for $5 \mathrm{keV}$ electrons scattered of $250 \mathrm{GeV}$ protons moving in the opposite direction. For this example, the energy of the electrons in the proton rest frame is $157 \mathrm{MeV}$.

charge, $E$ and $p$ the energy and momentum of the electron in the frame of the nucleus and $\theta$ the electron scattering angle in that frame. The first term is the classical Rutherford cross section and the two bracketed terms come from the $1 / 2$ spin of the electron and the nuclear recoil respectively. A small correction for the nuclear magnetic moment has been neglected. Another correction that has been neglected is the one for bremsstrahlung, which can be significant. A more complete theoretical treatment will probably be required to make good quantitative predictions, especially for even higher energy protons or ions.

Values of this cross section are computed at small angular intervals and then relativistic transformations to the laboratory frame of the cross sections, the angles and the energies lead to results such as plotted in Fig. 1. Such plots are useful for rough estimates of counting rates, but detailed comparisons are difficult due to the complicated nature of the spiraling electron trajectories in our particular application (see next section).

For the same example, the laboratory angle is plotted in Fig. 2 as a function of the angle in the proton frame of reference, both angles being shown in this case with respect to the original electron propagation direction. We see that this is a rather extreme example of relativistic beaming, also referred to as the "headlight effect." Electrons scattered forward in the proton frame at angles larger than $\sim 0.1$ degrees appear in the laboratory at angles larger than 90 degrees, i.e. they are backscattered.

\section{RHIC ELECTRON LENS BEAM ALIGNMENT}

The partial compensation of the head-on beam-beam effect in RHIC is necessary for mitigating the limit imposed by this effect on the achievable proton-proton beam luminosities [11]. Electron lenses (e-lenses) [15-17] consisting of low energy (in our case $\sim 5 \mathrm{keV}$ ), high intensity ( 1 A), magnetized electron beams [18], can provide the 


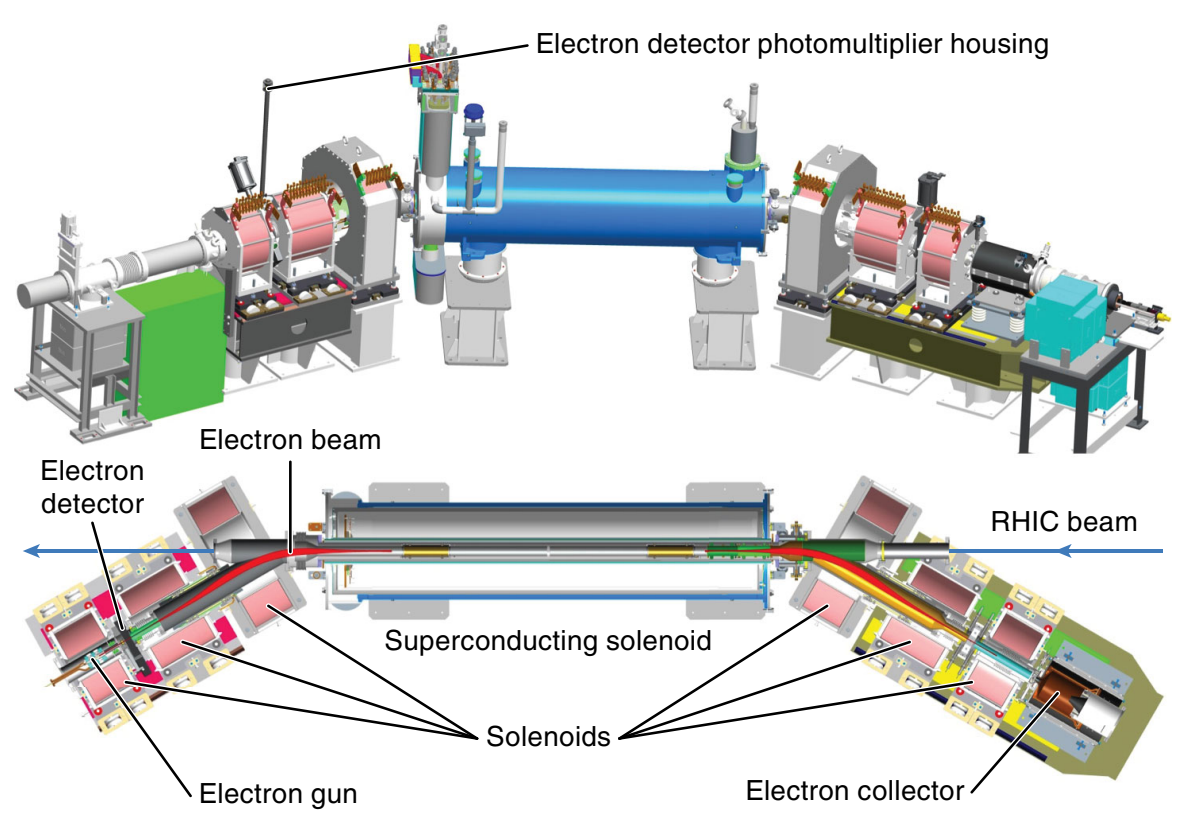

FIG. 3. Perspective and top views of one of the RHIC electron lenses [11,12], showing the backscattered electron detector location close to the electron gun. In reality, the light guide and photomultiplier tube are enclosed in a heavy and light-tight magnetic shield to protect the PMT from the stray fields of the nearby magnets.

precise nonlinear focusing properties necessary to effect such compensations. A schematic view of the two electron lenses that are being used for this purpose in RHIC is shown in Fig. 3.

The precise alignment overlap of the electron and ion beams is an important prerequisite for achieving maximum compensation and for avoiding deleterious effects on the proton beam emittance [19-21]. Over the $2.4 \mathrm{~m}$ interaction region in the up to $6 \mathrm{~T}$ solenoid, the centers of these, as small as $300 \mu \mathrm{m}$ rms wide beams, need to be separated by less than $50 \mu \mathrm{m}$. The precision achievable with the installed beam position monitoring system is not sufficient for ensuring this result, especially in view of electronic offsets that are not identical for electrons and ions [22,23]. Besides, in order to generate beam position monitor (BPM) signals, the dc electron beam needs to be modulated and this modulation may affect electron beam stability during operation. As mentioned above, electrons in the electron beam, backscattered by the relativistic protons can provide a signal proportional to the electron-proton luminosity that can be used to maximize the overlap of the two beams [10].

The lensing effect of an e-lens on the relativistic protons is due to the macroscopic electric and magnetic fields produced by the Gaussian-shaped electron beam. In other words, it is the collective long-range Coulomb interaction of the electrons with individual ions that affects the trajectory of these ions. The vast majority of the electron trajectories are only slightly affected transversely [24] since their trajectories are confined by a strong solenoid magnetic field. There is, however, a finite probability for ion-electron collisions with impact parameters that are so small as to produce a significant electron scattering angle, imparting at the same time considerable momentum and energy to the scattered electrons. Large scattering angles, correlated with high energies, result in energetic electrons spiraling backwards (towards the electron gun) along the magnetic field lines. Some of these backscattered electrons are intercepted and counted by a scintillation detector placed in air, behind a thin vacuum window.

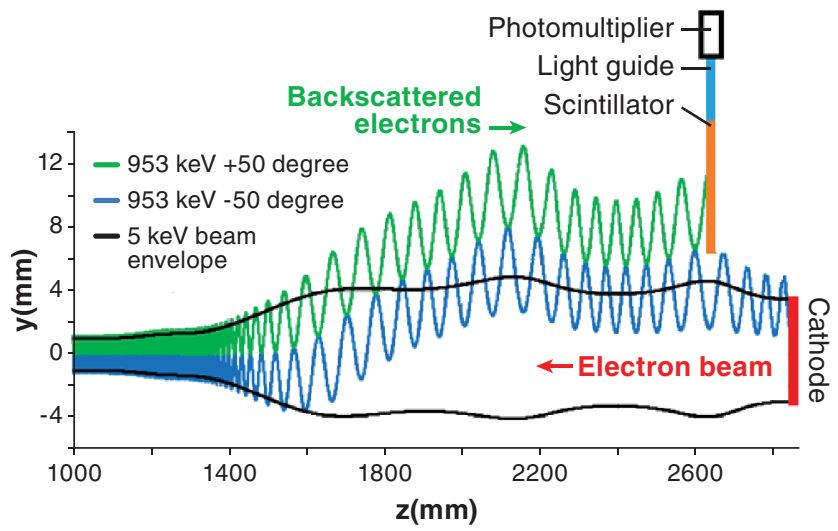

FIG. 4. Computer simulated trajectories (blue and green curves) of two scattered electrons generated inside of the $6 \mathrm{~T}$ solenoid (see text). Only the first $200 \mathrm{~mm}$ of this $2400 \mathrm{~mm}$ long superconducting solenoid is included at the left (from $\mathrm{Z}=$ $1000 \mathrm{~mm}$ to $\mathrm{Z}=1200 \mathrm{~mm}$ ). Three weaker solenoids (not shown) guide the electron beam from the cathode towards the $6 \mathrm{~T}$ region (see Fig. 3). 


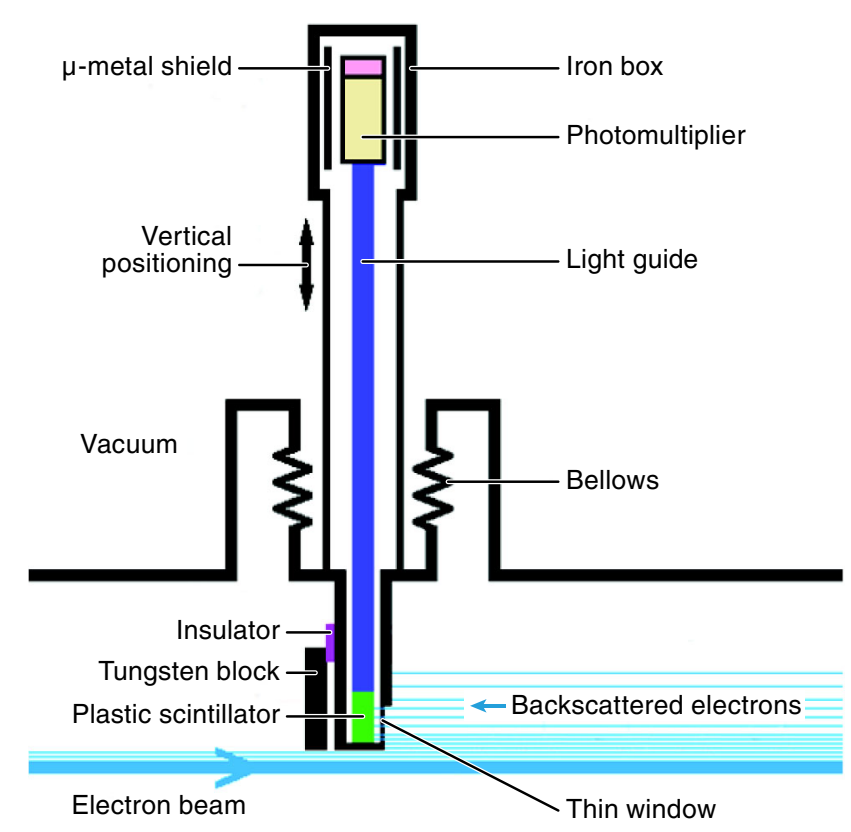

FIG. 5. Not-to-scale schematic of the eBSD scintillation detector and its housing. Electrons backscattered by the relativistic ion beam reach the plastic scintillator after traversing a thin titanium alloy vacuum window. The light from the scintillator is converted to electrical signals by means of a well shielded photomultiplier tube. A tungsten block protects the back of the detector cavity from accidental heating by the electron beam.

Figure 4 shows the simulated projected trajectories of two electrons backscattered by $250 \mathrm{GeV}$ protons in a $6 \mathrm{~T}$ solenoid at angles of $\pm 50^{\circ}$, one upwards and the other one downwards. As the electrons spiral towards the detector, the radii of their trajectories grow as they encounter lower fields. The upward drift of the trajectory envelopes of the backscattered electrons is due to the horizontal bend in the field [25]. The higher energy of the scattered electrons as compared to the electrons in the primary beam makes this drift appreciable for the latter, while it is negligible for the former. This upward drift of the scattered electrons is helpful in separating the electron trajectories from the primary electron beam, thus facilitating their detection.

Each RHIC electron lens is equipped with an eBSD consisting of a small plastic scintillator $(7.4 \times 7.9 \times$ $20.6 \mathrm{~mm}^{3}$ ) attached to a $1.2 \mathrm{~m}$ long light guide leading to a small magnetically shielded photomultiplier (PMT) tube (Hamamatsu R3998-02) [26]. The signals from this PMT reach the instrumentation rack through a $\sim 90 \mathrm{~m}$ long, $50 \Omega$ coaxial cable and are amplified and connected to a fast discriminator, the output pulses of which are used to determine the counting rates. The long light guide is necessary to keep the PMT far enough from the adjacent magnets so as to enable adequate shielding. This scintillation detector assembly is mounted in air in a vertical shaft, at the bottom of which there is a $0.1 \mathrm{~mm}$ thick titanium alloy vacuum window facing the scintillator. The vertical position of the detector shaft can be selected so as to locate

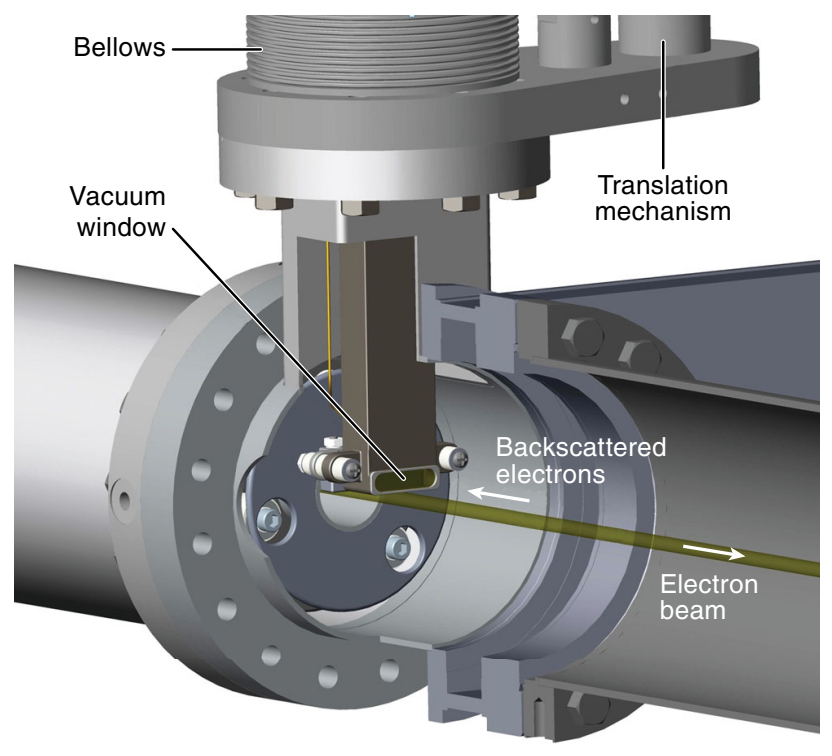

FIG. 6. Cutaway drawing of the detector housing, the vertical translation mechanism and the location of the $0.1 \mathrm{~mm}$ thick titanium alloy vacuum window in its lowest position. The area of this window is $25.4 \times 6.35 \mathrm{~mm}^{2}$. The vertical position can be changed by up to $25.4 \mathrm{~mm}$ away from the electron beam. That highest position was the one used for most measurements to minimize excessive counting-rate issues.

the bottom of the scintillator at any position from 1 to $25.4 \mathrm{~mm}$ from the edge of the primary electron beam. This position adjustment can be used as an intensity range selector. An insulated tungsten block $\left(35 \times 4.9 \times 7.6 \mathrm{~mm}^{3}\right)$ with current detection provides some protection against electron beam heating, should the position interlock and limit switch fail. Such a failure actually occurred during commissioning and a RHIC vacuum failure was avoided thanks to the tungsten block even though indirect heating was sufficient to melt the scintillator. Since the scintillation detector was in air, it was easily replaced.

A not-to-scale schematic and a cutaway drawing of the detector housing are shown in Figs. 5 and 6 respectively.

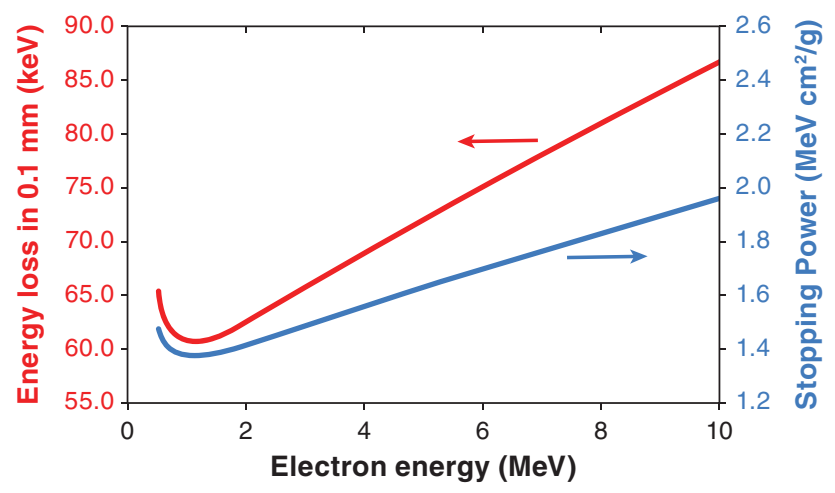

FIG. 7. Stopping power (blue) [27] and calculated energy loss (red) in titanium as a function of electron energy. 


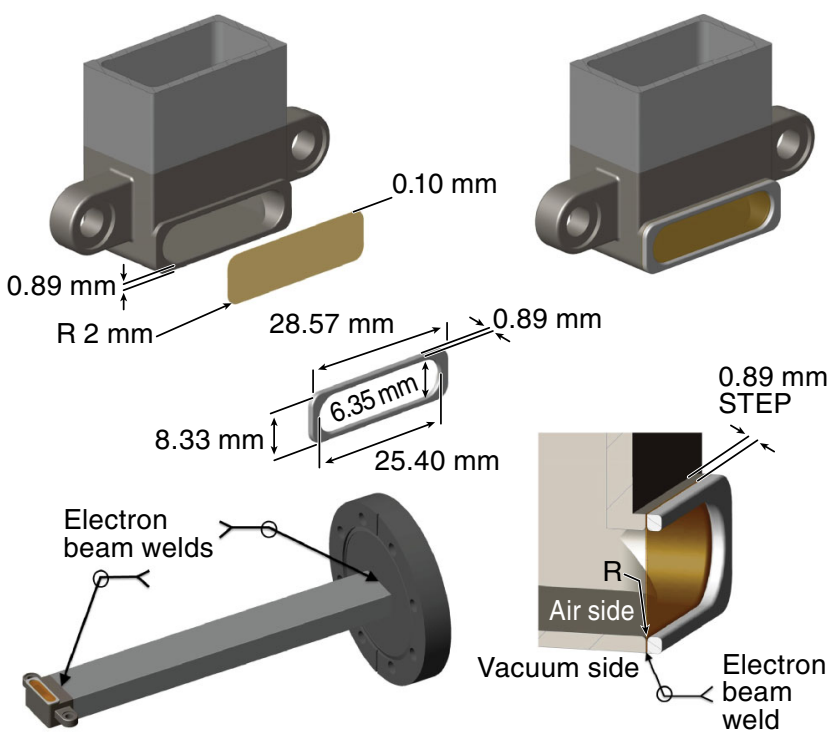

FIG. 8. Design and fabrication details of the cavity and the $0.1 \mathrm{~mm}$ thick titanium alloy window. The light guide is inserted in the cavity with the plastic scintillator facing the thin window. The electrons traverse this window from the vacuum outside of the cavity to the inside that is at atmospheric pressure.

The design and fabrication of the $100 \mu \mathrm{m}$ thick Ti-6Al-4V alloy window was a critical aspect of this project since the electron energy loss had to be minimized while guaranteeing the integrity of the RHIC vacuum system. Figure 7 shows the stopping power [27], and the calculated energy loss in a $0.1 \mathrm{~mm}$ thick titanium alloy window.

We see that the energy loss in a $0.1 \mathrm{~mm}$ thick titanium window is acceptable for electrons of a few hundred $\mathrm{keV}$ and up. The design and dimensions of the window and detector housing are shown in Fig. 8 and the corresponding stress analysis is presented in Fig. 9.

The detector cavity and the $0.1 \mathrm{~mm}$ thick window shown in Fig. 8 were fabricated using Ti-6Al-4V alloy which provided the desired strength and relatively small electron

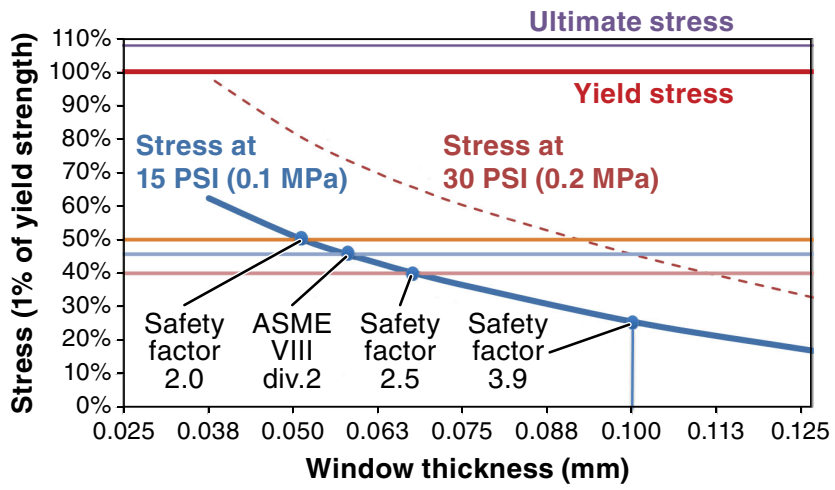

FIG. 9. Finite element stress analysis results for the $25.4 \times$ $6.9 \times 0.1 \mathrm{~mm}^{3}$ Ti-6Al-4V alloy window. There is a safety factor of 3.9 at atmospheric pressure. The window was pressure tested up to three atmospheres without bursting. energy loss (see Fig. 7). The stress analysis shown in Fig. 9 as well as pressure tests proved that the safety factor is larger than 3.

\section{COMMISSIONING WITH GOLD AND ${ }^{3} \mathrm{He}$ BEAMS IN RHIC}

The commissioning of the eBSDs was started during the $2014100 \mathrm{GeV} /$ nucleon gold-gold run. The first proof-ofprinciple horizontal and vertical beam separation scans are shown in Fig. 10.

The measured widths are both about $25 \%$ larger than the sums in quadrature of the gold and electron beam widths. This small discrepancy could be due to residual angular misalignments, to small ion beam motions or to errors in the gold beam beta function estimates.

Soon after obtaining these results, a beam alignment optimization system was implemented based on automatically maximizing both the eBSD counting rates as a function of horizontal and vertical positions and angles. This system is based on a program (LISA) [28] that was developed many years ago and used since then to maximize the ion-ion luminosities for the RHIC experiments by
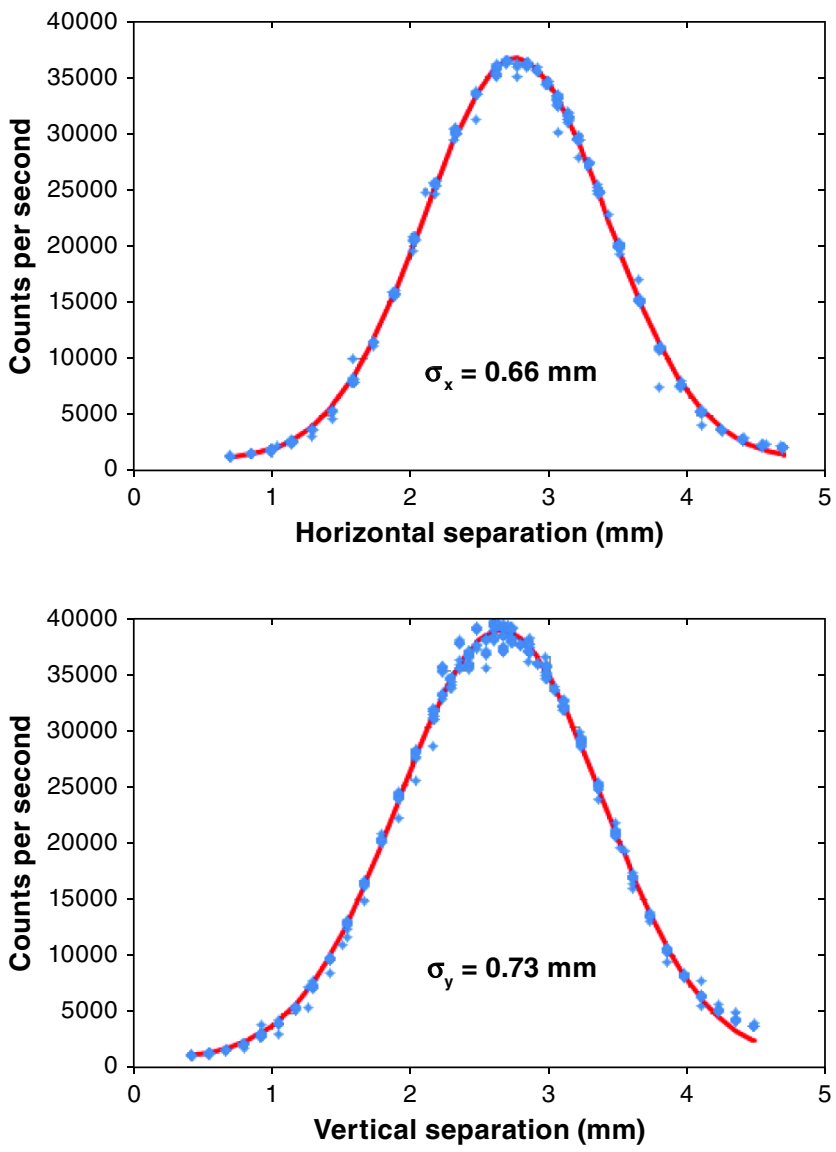

FIG. 10. Horizontal and vertical beam separation scan obtained by steering the $5 \mathrm{keV}$ electron beam with respect to the $100 \mathrm{GeV} /$ nucleon gold beam. 


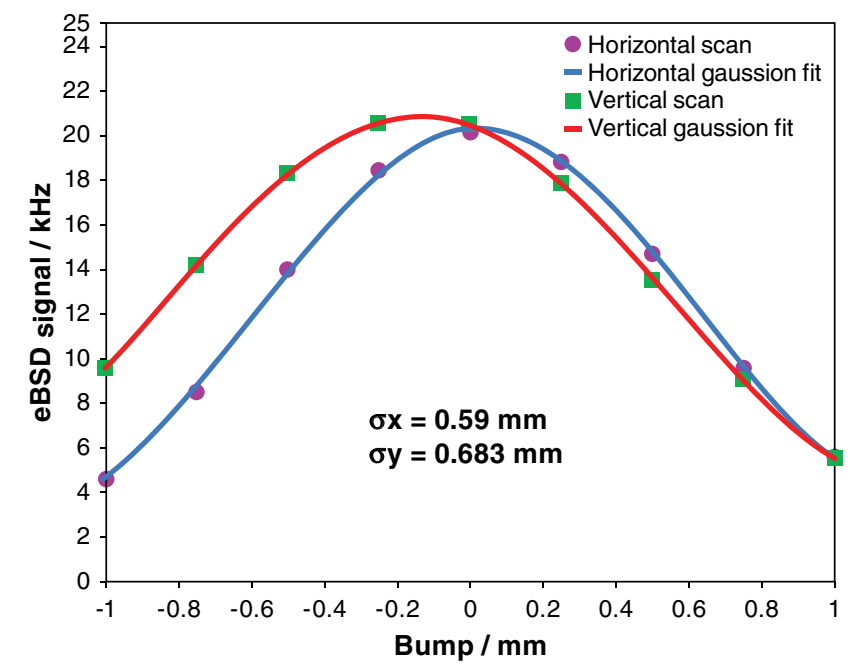

FIG. 11. Manual beam separation scans obtained by stepwise steering the ${ }^{3} \mathrm{He}$ beam with closed bumps utilizing part of the algorithm developed for the automated alignment optimization system based on the LISA program [28].

maximizing the coincidence rates from the zero degree calorimeters (ZDCs) [29].

After the gold run was completed, there was a brief opportunity for commissioning the eBSD system with a ${ }^{3} \mathrm{He}$ beam. This was important since gold scattering cross sections are much larger than the cross sections for protons, and therefore the gold beam tests were not representative of the situation with protons. The cross section for ${ }^{3} \mathrm{He}$ is $\sim 4$ times larger than for protons [see Eq. (1)] but the beam intensity was smaller. The counting rates for ${ }^{3} \mathrm{He}$ were similar to the ones expected for protons. Horizontal and

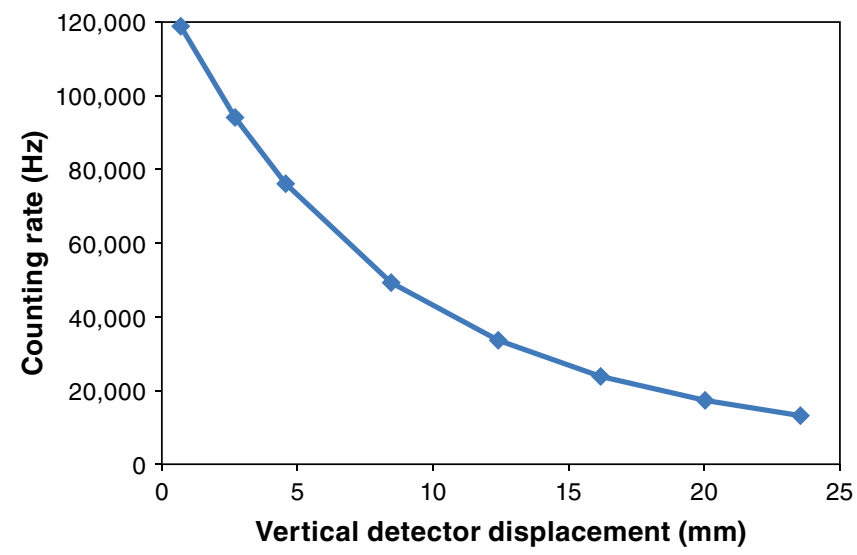

FIG. 12. Counting rate as a function of the vertical displacement of the eBSD detector. These results were obtained with a $100 \mathrm{GeV} /$ nucleon ${ }^{3} \mathrm{He}$ beam consisting of 93 bunches with $4.7 \times 10^{10}$ ions per bunch and a $6 \mathrm{keV}, 88 \mathrm{~mA}$ electron beam. The zero of the displacement scale corresponds approximately to the center of the detector being $5 \mathrm{~mm}$ above the center of the electron beam. The operating point was the rightmost point on the chart, at $24 \mathrm{~mm}$. The counting-rate slope at that point is approximately $6.8 \% / \mathrm{mm}$. vertical separation scans are shown in Fig. 11. These data are from a manual LISA eBSD scan obtained by displacing the gold beam by means of a set of steering correctors forming closed horizontal and vertical bumps.

During the ${ }^{3} \mathrm{He}$ run the vertical positioning mechanism was utilized for the first time since the counting rates with gold had always been so large that the fully retracted position had to be used. Figure 12 shows the counting rate as a function of detector position for a $100 \mathrm{GeV} /$ nucleon ${ }^{3} \mathrm{He}$ beam consisting of 93 bunches with $4.7 \times 10^{10}$ ions per bunch and a $6 \mathrm{keV}, 88 \mathrm{~mA}$ electron beam.

\section{UTILIZATION OF THE EBSDS DURING THE 2015 RHIC POLARIZED PROTON RUN}

During this two-month run, the eBSDs were used routinely as the main alignment and monitoring tools for the electron and proton beam overlap in both electron lenses, without any system failures.

To ensure optimal pulse height discriminator settings, rejecting low amplitude noise while minimizing any impact of gain shifts, a pulse-height analysis system was implemented shown schematically in Fig. 13.

Figure 14 shows pulse height spectra and selected discriminator settings. The pulse-height resolution is poor, mainly due to the small photon collection efficiency through the thin, $1.2 \mathrm{~m}$ long light guide. However, excellent signal-to-noise ratios are achieved by adequate selection of the discriminator setting.

The stability of the system was such that only one slight readjustment was performed during the entire period. By the end of the run, there was a $\sim 12 \%$ pulse height reduction measured with a precise light pulse generator

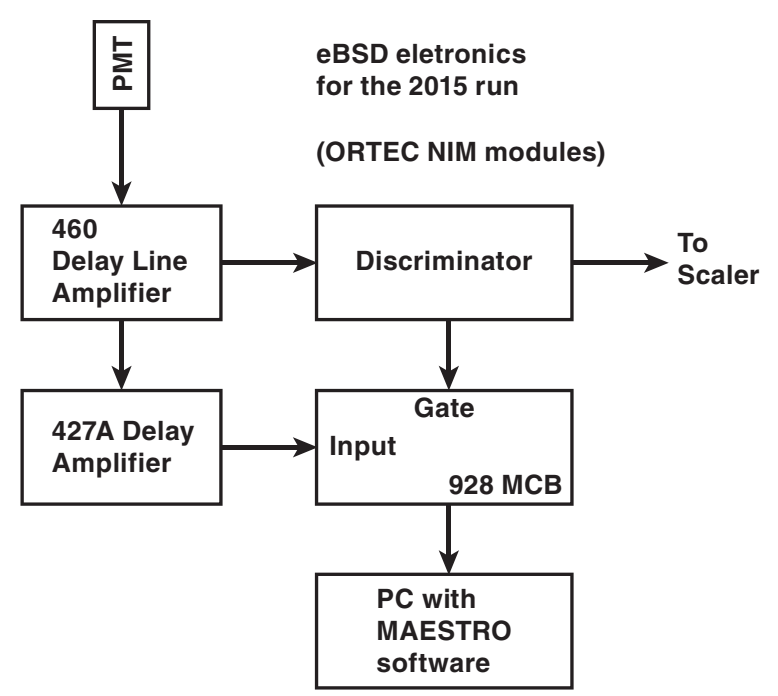

FIG. 13. Schematic of the pulse height analysis system used to optimize the discriminator setting. The multichannel analyzer (MCA) used here consists of an Ortec multichannel buffer (MCB) connected to a computer running the Maestro analysis and control software [30]. 

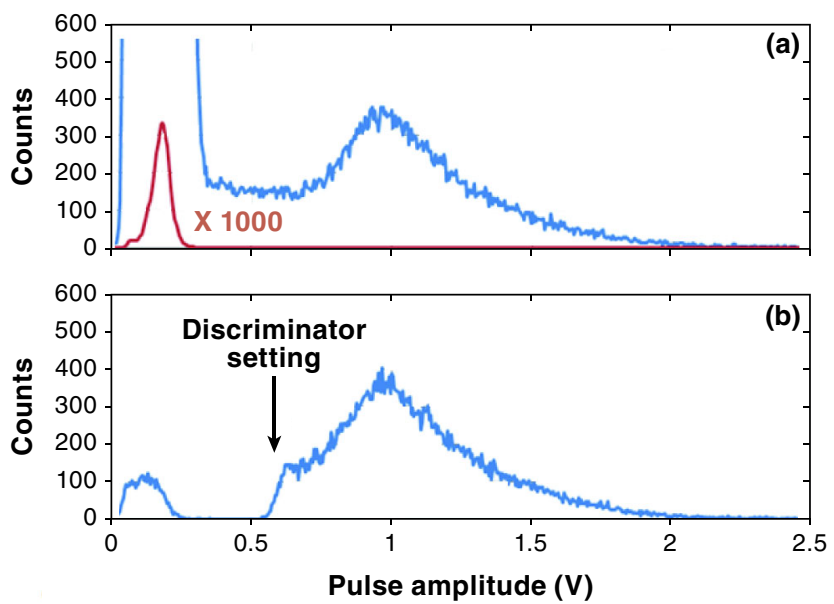

FIG. 14. Pulse height spectra from the scintillation counter used in one of the RHIC electron lenses. In the upper spectrum (a) all pulses are accepted, while in the lower one (b), pulse amplitudes below the discriminator setting are rejected. The discriminator effectively suppresses the high intensity low amplitude noise (see the red curve, which is the original histogram plotted with a scale change of a factor 1000). The small remaining peak to the left in (b) may be an artifact of the pulse height analyzer gating system.

[31]. This slight pulse height reduction is illustrated in Fig. 15 which shows screen shots of counting rates as a function of pulse height from the pulse height analyzer program, MAESTRO [30]. All settings were identical and the pulse height of the light pulser peak is reduced by about $12 \%$ after two months of continuous use. This reduction may indicate slight radiation damage of the $1.2 \mathrm{~m}$ long light pipe and/or of the fiber carrying the light to its far end. It would not reflect any reduction in the scintillation

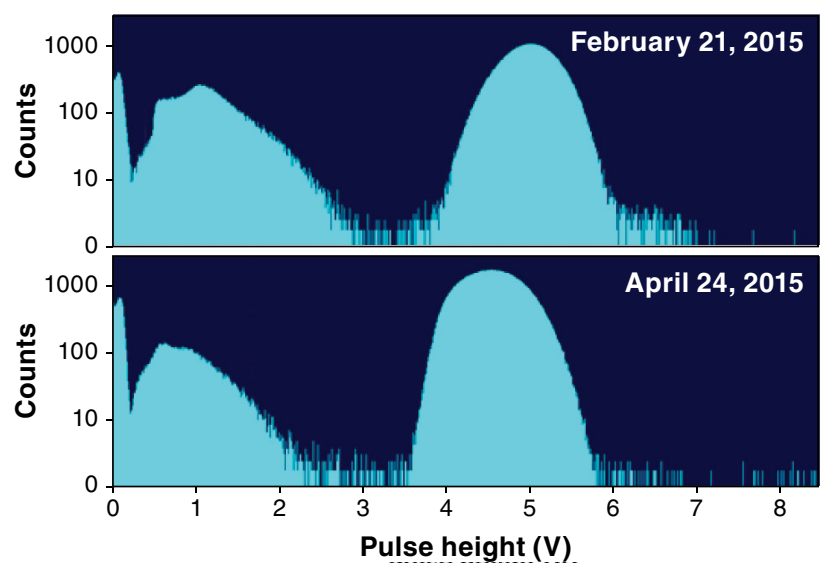

FIG. 15. Pulse height spectra screen shots obtained with the pulse-height analyzer software [30]. The logarithm of counting rates is displayed as a function of pulse heights. The peaks to the right were obtained using identical settings of a precision light pulse generator. The slight shift of this peak indicates a $~ 12 \%$ light transmission loss due perhaps to slight radiation damage of the $1.2 \mathrm{~m}$ long light guide or of the fiber carrying the light signal to the far end of the light guide.
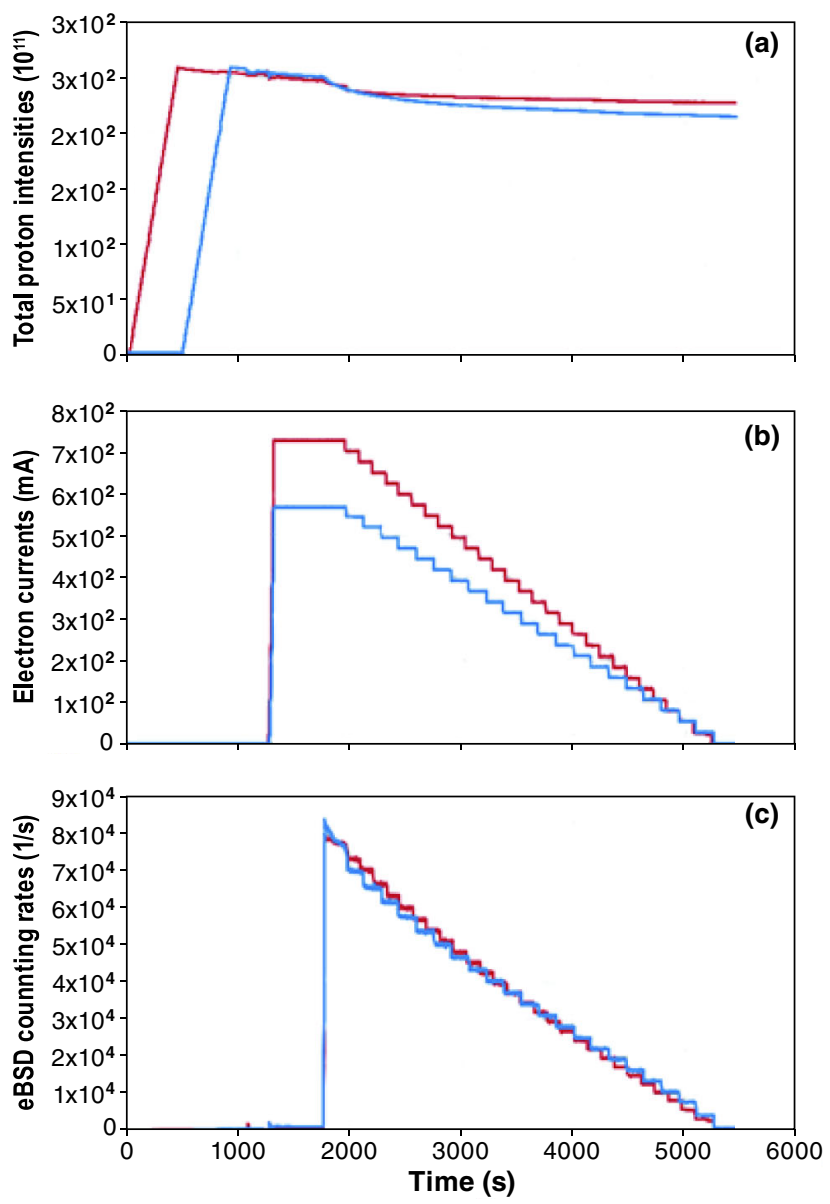

FIG. 16. The total proton intensities (a), RHIC electron lens electron current intensities (b), and corresponding eBSD counting rates (c) are shown as a function of time. The electron beam intensities are reduced in steps to optimize the benefits of the RHIC electron lenses [11]. The two colors indicate the results from each of the two RHIC rings.

efficiency. Rather than using this light pulser as a reference, it would have been better to install a very weak radioactive source for continuous, end-to-end gain verification. Modest reductions in pulse height can be easily compensated by adjusting the PMT high voltage. If necessary, the detector assembly can be easily replaced.

The optimization of the beam alignment was largely automated by using the LISA algorithm described above [28]. To simplify the angular adjustments, the algorithm was modified, so as to rotate the beams around the centers of the respective lenses rather than around the protonproton crossing point which is $3.3 \mathrm{~m}$ away from the center of the lenses. No interactions occur at this crossing point because the two beams are at different heights being separated by $10 \mathrm{~mm}$, or approximately 20 sigmas.

During this run, compensation with close to maximum electron current was only used at the beginning of each store and was then reduced in steps as shown in Fig. 16. This optimized compromise provided the best integrated 

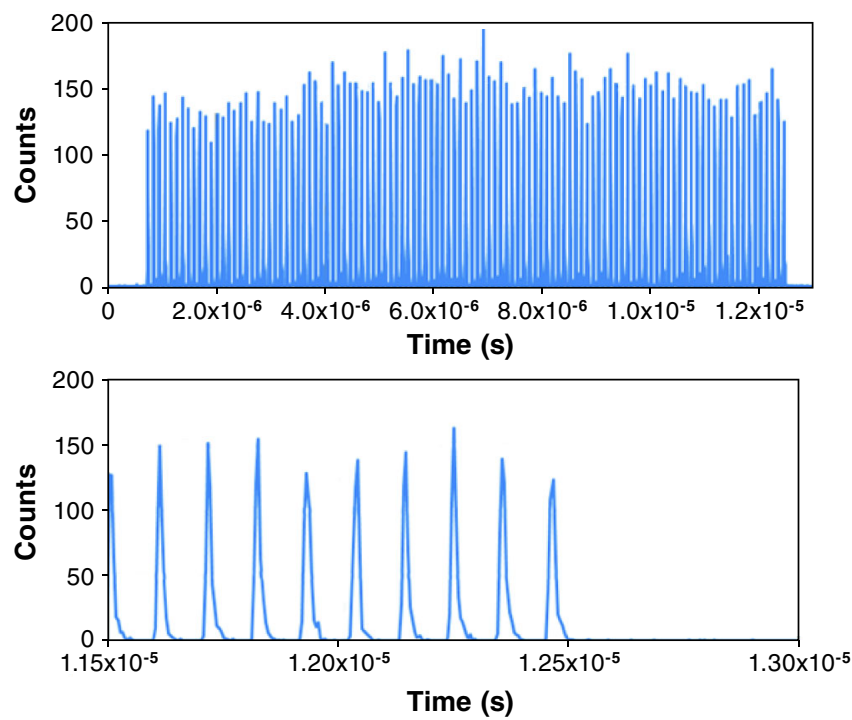

FIG. 17. Time-of-flight spectra of electrons generated by the interaction of the relativistic proton beam with the residual gas. Data were accumulated for 5 minutes due to the low counting rates. The top chart shows the RHIC 111-bunch pattern and portions of the abort gaps preceding and following that pattern. The bottom chart is an expanded view of the last ten bunches to show the asymmetric shape of the peaks (see text).

luminosity by utilizing the electron lenses when most necessary for compensation, while minimizing their impact on beam lifetime and emittance [11].

During this period, a system that sorts the eBSD signals according to their arrival time was tested [10]. For this purpose, time digitizers [32] were started by the eBSD signals and stopped by a signal synchronized to the RHIC revolution frequency. While this system was not utilized, we mention it here because it may be used in the future and may also be of interest for other applications (see the next section).

Figure 17 shows a time spectrum obtained with signals from the proton beam scattering electrons from the residual gas. Variations in count rate from peak to peak are consistent with variations in the individual bunch intensities. The 111-bunch structure representing one turn (Trev $=12.8 \mu \mathrm{s}$ ) is shown at the top, and the last ten bunches followed by part of the abort gap are shown at the bottom. The fast rising part of the peaks represents electrons arriving early which tend to originate closer to the gun, while the right side of the peaks, with a gentler slope, corresponds to later electrons from the other end of the interaction region. This effect could, in principle, be used as an aid for angular tuning of overlapped electron and proton beams.

\section{SYSTEMATIC ERROR ESTIMATES}

While experimental results with proton-proton collisions [11] are compatible with having achieved perfect overlap
TABLE I. Beam parameters during the ${ }^{3} \mathrm{He}$ tests.

\begin{tabular}{lccc}
\hline \hline Location & & $\begin{array}{c}\text { Interaction } \\
\text { region }\end{array}$ & $\begin{array}{c}\text { eBSD } \\
\text { detector }\end{array}$ \\
\hline Magnetic field & $(\mathrm{T})$ & 4.00 & 0.25 \\
e-beam rms size & $(\mathrm{mm})$ & 0.375 & 1.50 \\
${ }^{3}$ He-beam vertical & $(\mathrm{mm})$ & 0.32 & \\
$\quad \begin{array}{l}\text { rms size } \\
{ }^{3} \text { He-beam horizontal rms size }\end{array}$ & $(\mathrm{mm})$ & 0.46 & \\
\hline \hline
\end{tabular}

between the electron and proton beams, possible deviations are difficult to estimate from these measurements. We explore in this section to what extent maximizing the eBSD counting rates may result in imperfect overlap. We identify two sources of systematic errors, one in the horizontal and one in the vertical alignment, and we estimate the magnitude of these errors with simulations for the specific example of the ${ }^{3} \mathrm{He}$ tests for which we have the necessary data. Table I lists the relevant parameters.

For the horizontal alignment, there is an obvious bias due to the fact that at the entrance and exit of the electronion beam overlap region, the electron beam deviates from the straight trajectory on its way from the electron gun and to the electron collector. As shown in Fig. 18 there are regions at both ends of the overlap region where the electron beam becomes larger and curves away from the ion beam trajectory. Backscattered electrons from these regions will make an asymmetric contribution to the counting-rate curve when scanning one beam across the other.

In Fig. 18(c) we show a magnified view of the area where this asymmetry arises at the electron collector side of the interaction region. The electron beam transport is symmetric around the center of the solenoid since the magnetic field is symmetric [see Fig. 18(a)]. In Fig. 18(c) we also show a \pm 1 sigma ion beam profile centered with respect to the electron beam in the solenoid. We estimate the relative counting-rate variation as a function of offset by computing the convolutions of these two beams (assumed to be Gaussian) as a function of offset in $0.1 \mathrm{~mm}$ steps. The result is shown in Fig. 19, and compared to the simulated counting-rate profile in the absence of the asymmetric contribution.

The counting-rate asymmetry as a function of horizontal beam offset is clearly visible. The peak position was calculated with a quadratic fit over a $\pm 0.2 \mathrm{~mm}$ range, following the procedure used by the automatic adjustment software [28]. The peak offset is $0.018 \mathrm{~mm}$ for this example where the rms widths of the electron and ion beams are 0.375 and $0.46 \mathrm{~mm}$ respectively. This shift is not expected to have any measurable consequences upon the e-lens performance in this case. If much larger deviation should occur in other situations, corrections could be computed and applied. 


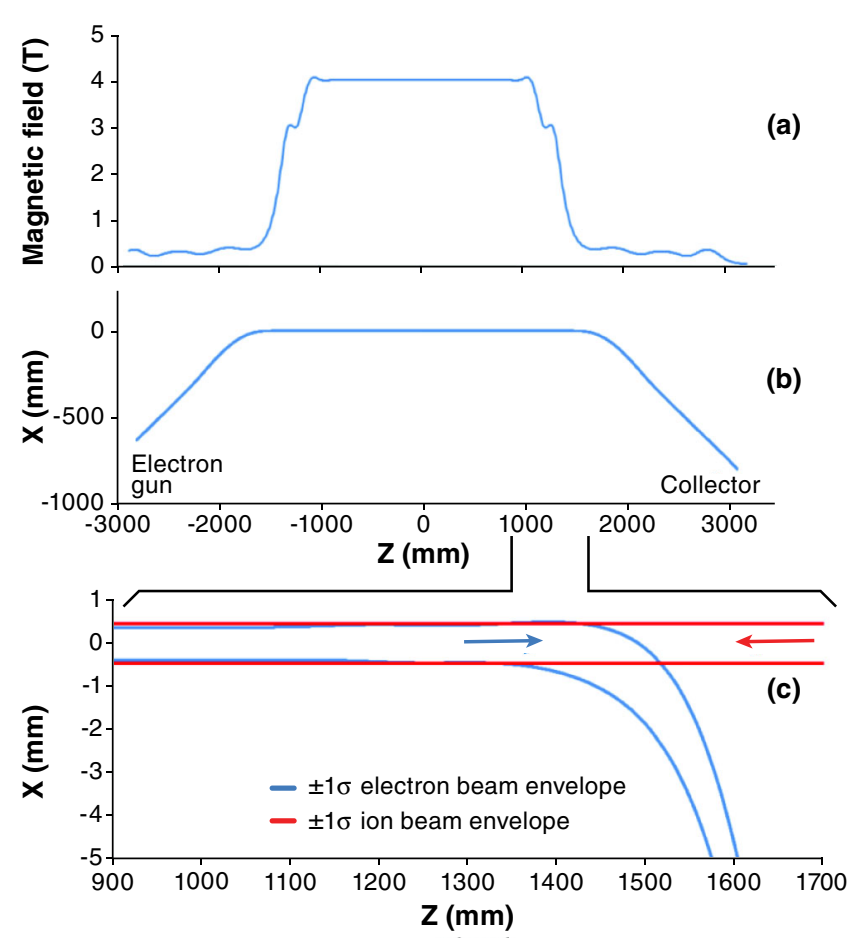

FIG. 18. Magnetic field profile (a) and electron beam trajectory (b) starting at the electron gun and ending at the electron collector. In the magnified view (c) we show the \pm 1 sigma electron beam and ion beam profiles in the region where the electron beam starts curving away on its way to the collector.

In the vertical direction, there is a bias introduced by the detection efficiency dependence on the vertical position of the Coulomb interaction point. In other words, a backscattered electron originating from a point located at some distance below the axis common to the electron and ion beams will have a slightly different detection probability compared to another electron originating at a point located above that axis. This effect could be measured by vertically

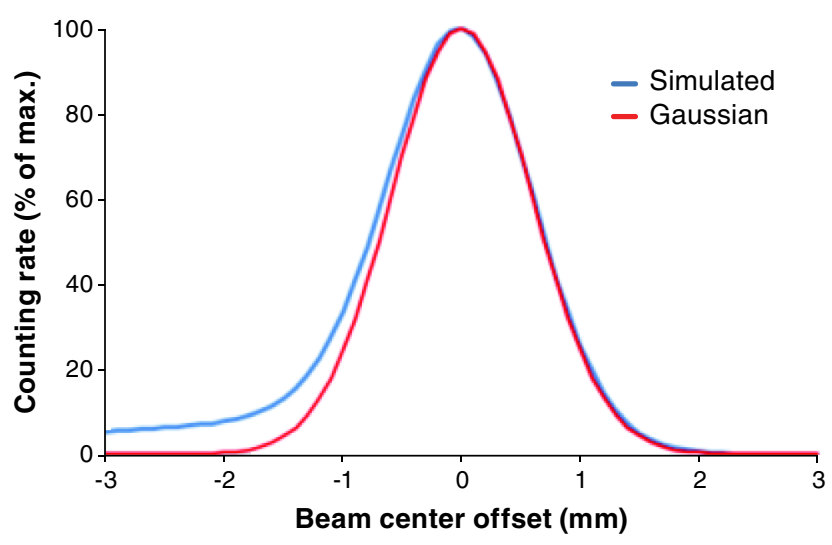

FIG. 19. Simulated relative counting rates as a function of horizontal electron-ion beam offset for the case illustrated in Fig. 18. Also shown (in red) is the ideal convolution of the two Gaussian beams in absence of the asymmetric contributions. displacing the interaction region, i.e. both the electron and the ion beams together, and monitoring associated counting-rate changes. While this approach may be attempted in the future, we will here arrive at an estimate based on the measured counting-rate dependence on the vertical position of the detector shown in Fig. 12. The slope of this curve at the operating position at $24 \mathrm{~mm}$ is $\sim 6.8 \%$ per $\mathrm{mm}$. This detection efficiency slope at the detector location can be converted to an equivalent efficiency slope in the interaction region. To that effect, we take into account the adiabatic invariance of the flux through the electron orbits [25] which leads to similar projections of the electron orbits onto planes perpendicular to the field at the detector position and in the interaction region. These similar projections differ by a scale factor equal to the ratio of the square roots of the magnetic field strengths at these two locations. In the present case that scale factor is 4.0 (see Table I). That in turn means that the slope of the detection efficiency translated to the interaction region will be $6.8 \times 4=27.2 \% / \mathrm{mm}$.

The counting rate as a function of ${ }^{3} \mathrm{He}$ beam position will be the usual convolution of two Gaussians, but modified here by the efficiency which we approximate as a linear function of the position with the slope $27.2 \% / \mathrm{mm}$ obtained above. Using this value for the parameter $\mathrm{k}$, and the vertical rms beam sizes $\sigma_{\mathrm{He}}$ and $\sigma_{e}$ from Table I, we use Eq. (2) to calculate values proportional to the eBSD counting rate as function of the He beam position:

$$
\begin{aligned}
N\left(y_{\mathrm{He}}\right)= & \int_{-\infty}^{\infty} \exp \left[-\frac{y_{e}^{2}}{2 \sigma_{e}^{2}}\right] \times \exp \left[-\frac{\left(y_{e}-y_{\mathrm{He}}\right)^{2}}{2 \sigma_{\mathrm{He}}^{2}}\right] \\
& \times\left(1+k y_{e}\right) d y_{e} .
\end{aligned}
$$

The approximation that was made in this estimate is neglecting the small variations in vertical drift corresponding to variations in longitudinal electron velocity. These variations are small because of electron momentum conservation and because of the small angles between the electron trajectories and the magnetic field lines in the region of the bend. For the example shown in Fig. 4, these angles are around $\sim 8^{\circ}$. The result is an approximately Gaussian curve of rms width $\sigma \sim \sqrt{\sigma_{e}^{2}+\sigma_{\mathrm{He}}^{2}}$, with its maximum displaced by $0.028 \mathrm{~mm}$. This is less than $10 \%$ of the rms widths of either beam. A correction is not necessary in this case.

In other situations, in particular for beams of much larger widths, corrections could be computed or measured as outlined above, and applied by introducing a position correction after maximizing the counting rate.

\section{FUTURE POSSIBILITIES}

In this section, we briefly present a few preliminary ideas on how the detection of energetic scattered electrons could 
be used in other beam diagnostic applications. These ideas are based on the previous extensive use of electron beams as diagnostic tools, documented in the literature, and on the results and experience gained during the design, implementation and application of this new approach.

\section{A. eBSDs used with hollow electron beams as possible halo monitors and beam alignment tools}

Hollow electron beams have been tested as collimators or halo monitors in the Tevatron [33-36] and are being considered as an option to complement the LHC collimation system [13]. Here, we suggest that the backscattered electrons from the proton electron collisions could be detected and used for halo diagnostics and for centering the proton beam [37].

The arrangement would be similar to the BNL electron lenses, but additional thought is required to determine the best way to merge the beams and to separate them after the interaction region without producing unduly large background counting rates in the detectors.

A schematic illustration of the interaction between the halo protons with the electrons in the hollow beam is shown in Fig. 20. In reality, two or four equidistant detectors surrounding the beam would probably be used. The core of the proton beam will also produce energetic electrons by collision with the atomic electrons of the residual gas. This is the principal source of background and will determine the ultimate sensitivity for halo detection. For a rough

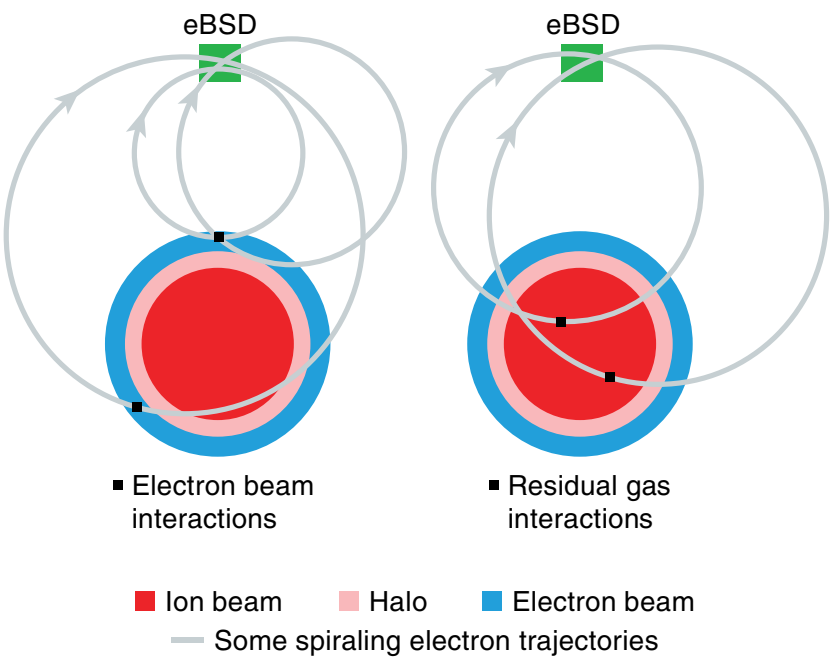

FIG. 20. Schematic illustration of the potential use of eBSDs for monitoring the halo of an intense ion beam. The beam propagates in a direction perpendicular to the plane of the figure. The electrons generated by the interaction of the ions with the hollow electron beam spiral along the magnetic field towards the detector generating a counting rate approximately proportional to the overlapped halo intensity (left). The ion beam interacting with the residual gas produces a background (right). Excellent vacuum is required to minimize this background (see text). estimate of this background we note that a $4 \mathrm{keV}$ electron current density of $1 \mathrm{~A} / \mathrm{mm}^{2}$ has an electron density equal to the electron density in $2.15 \times 10^{-6}$ Torr of $\mathrm{H}_{2}$ at room temperature. For an example of a round Gaussian ion beam of rms width $\sigma$ we conclude that for a hollow, $1 \mathrm{~A} / \mathrm{mm}^{2}$ electron beam extending from $4 \sigma$ to $5 \sigma$ the signal-tobackground ratio would be approximately 1 if the vacuum is $7 \times 10^{-10}$ Torr at room temperature. A better vacuum and/or a more intense electron beam will improve this signal-to-background ratio. Exceptionally good vacuum in a room-temperature chamber should be achievable in a beam pipe section pumped by a continuous longitudinal cryopumped antechamber as mentioned for example in Ref. [38]. If that is impractical, a nonevaporable getter (NEG) coated and activated beam pipe would be excellent too. The use of a warm chamber with an adjacent distributed cryopump is appealing since the quantity of interest to reduce the background is the gas density which, at constant pressure, is inversely proportional to the absolute temperature.

A technique that can be used to extend the dynamic range of these measurements involves modulating or pulsing the electron beam. Depending on counting statistics, results could be obtained with signal to background ratios as small as a few percent.

Figure 21 shows schematically the topology of three possible implementations. The first one seems elegant and appealing but it may be difficult to implement an annular cathode surrounding the proton beam. The second one has the same geometry as the existing electron lenses, but the ion beam intersects the electron beam on the collector side producing unwanted background. Finally, the third option solves these problems by locating the gun with the annular cathode to one side, and uses an annular collector surrounding the proton beam which should be possible to implement. This appears to be a viable option for a system that could be used as a halo monitor and as a beam alignment tool.

Aligning electron "wires" proposed as LHC long-range beam-beam compensators [39] may be achieved in a similar way, and without the complication of annular cathodes and collectors. The beams would be aligned by first overlapping them and then separating them by a known distance. If the electron beam remains partly in the halo of the proton beam, continuous monitoring would also become possible.

\section{B. Concept of a Coulomb scattering electron wire (CSeW) beam profile monitor}

Electron beams that are not collinear with the relativistic ion beam will also generate energetic scattered electrons that can in principle be used for beam diagnostics. An example is schematically shown in Fig. 22. A ribbon shaped electron beam propagates at a right angle to the ion beam guided by a weak magnetic field (2B) that affects 


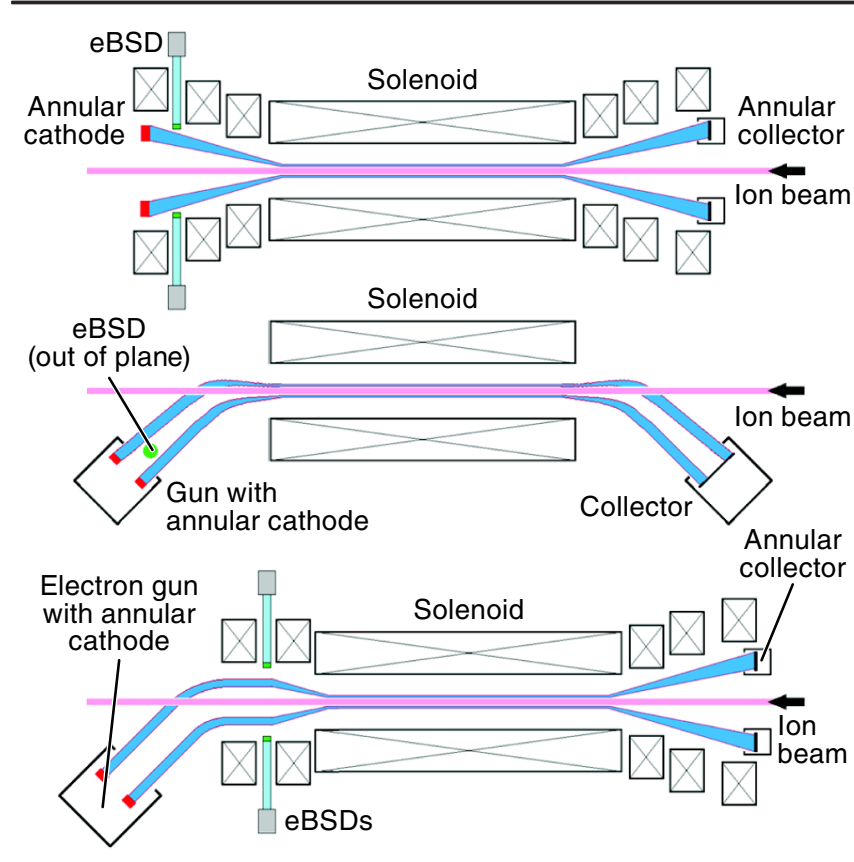

FIG. 21. Three possible configurations for using eBSDs for halo monitoring and beam alignment of hollow electron beam systems. The central long solenoid is the same strong field superconducting solenoid in each case, similar to the $6 \mathrm{~T}$ ones used in the Brookhaven RHIC electron lenses. The smaller and weaker room-temperature solenoids, indicated schematically by the small rectangles, guide the electron beam from the gun to the central solenoid and from there to the collector (see Fig. 3). Some of these guiding solenoids have been omitted for clarity. Only two eBSDs are shown in the bottom configuration, but there could be four at $90^{\circ}$ intervals for continuous halo monitoring and beam centering. The bottom configuration seems to be the most feasible one (see text).

the ion beam only slightly. This slight perturbation is compensated by the field B generated by the other two split solenoids.

The trajectories of the scattered electrons are bent in the field of the central split solenoid and some of them reach a scintillation detector through a vacuum window (not shown in the picture). Maximum intensity corresponds to optimal overlap. The beam profile can be explored by stepwise deflections of either the electron or the ion beam.

In contrast to conventional electron wire profile monitors [6-8], the profile is determined here by measuring the

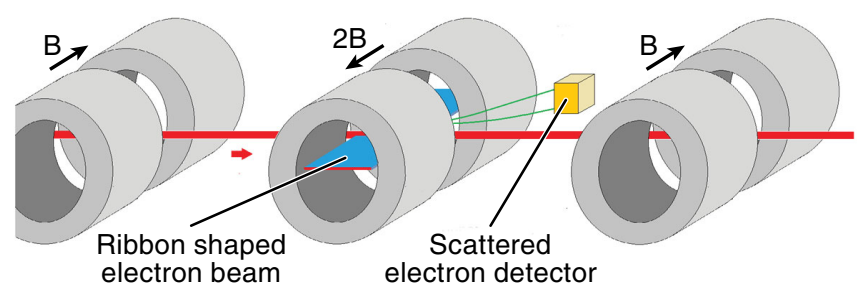

FIG. 22. Schematic illustration of a Coulomb scattering electron wire beam profile monitor (see text). counting rates of the scattered electrons and not by detecting the deflection of the electron trajectories, largely suppressed here by the transverse magnetic field. Potential advantages are that the measured profiles are largely independent of the beam intensity and that profiles are obtained directly as deflection-dependent counting rates. For relatively long bunches, the arrival time of the scattered electrons can be used to measure the time structure and head-tail position differences for each bunch. Two such systems, one horizontal and one vertical, would provide a rather complete characterization of the bunch through nondestructive measurements.

\section{Electrons scattered from residual gas atoms for beam diagnostics}

The interaction of particle beams with residual gas atoms and molecules is often used for measuring beam profiles such as in ionization profile monitors (see e.g. [40]) and fluorescence profile monitors (see e.g. [41]). Recently, a beam-gas vertexing technique [42] was used to characterize LHC beam properties by high precision tracking of particles from nuclear interaction with a small amount of gas injected into the vacuum chamber $[43,44]$.

We suggest here that detecting energetic scattered electrons is another good way to exploit the interaction with residual gas for beam diagnostic purposes. The cross sections for Coulomb interactions are orders of magnitude larger than for nuclear cross sections. Much less gas will therefore be required. As an example, we show a conceptual design for a beam position monitor for eRHIC [45], the proposed BNL ERL-based electron-ion collider. This is only one of several possibilities for the difficult task of monitoring the position of up to 24 side-by-side electron beams circulating in the same vacuum chamber and separated in time by as little as $2 \mathrm{~ns}$. As shown schematically in Fig. 23, two fast, position-sensitive channel-plate detectors detect the scattered electrons through sets of
SIDE VIEW

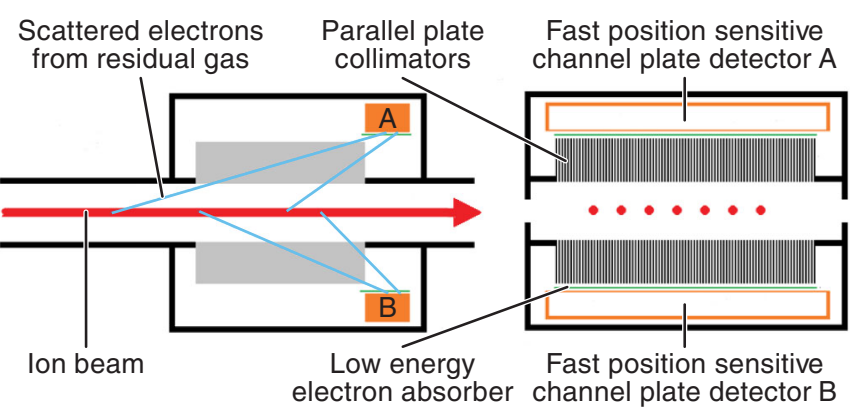

FIG. 23. Concept of one of the possible approaches to detect several side-by-side orbits in one of the fixed-field alternating gradient (FFAG) arcs of the electron-ion collider, eRHIC, which will be proposed as a successor to RHIC. Fast, position-sensitive channel-plate detectors respond to energetic electrons collimated by parallel plate collimators (see text). 
parallel plate collimators which are necessary to define the plane of the trajectories. Thin foils in front of the detectors stop low energy electrons from generating spurious signals. A second set of detectors and collimators, at right angles to the first one, could, in principle, be located in the same chamber. The detection by the channel plates is fast and the position resolution will be defined by the acceptance angle of the collimators.

\section{CONCLUSIONS}

Most instruments used for particle accelerator beam diagnostics are of the analog type where often small signals are transmitted through long cables, amplified and digitized. The few instances, when particle detecting and counting techniques can be used, offer the advantages of greater dynamic range and greater noise immunity which is particularly important in the harsh environment of high energy particle accelerators.

The detection of energetic electrons generated through Coulomb scattering by relativistic ions offers new possibilities for relativistic ion beam diagnostics. The fact that these electrons can traverse vacuum windows with relatively minor energy loss allows the convenient use of simple detectors such as scintillation detectors that are cumbersome to use in vacuum. The easy replacement of the detectors without disturbing the vacuum is also an important advantage.

We have shown here the successful application of such a system, used for the alignment of electron and ion beams in the RHIC electron lenses at BNL. A counting-rate dynamic range of about 5 orders of magnitude has been utilized so far. Given the fast response of the utilized scintillators and counting electronics, larger dynamic ranges are available. A likely improvement for future systems of this type will be the utilization of silicon photomultipliers which are not sensitive to magnetic fields. Shorter light guides and less magnetic shielding should simplify the design and improve the performance.

We also outlined ideas for other possible beam diagnostic applications based on energetic electrons produced by Coulomb scattering by relativistic ions.

\section{ACKNOWLEDGMENTS}

We are grateful to the RHIC operations crew for their expert assistance in the development, testing and implementation of this new system. We would like to thank the referees for many valuable suggestions. In particular, the idea of modulating the electron beam, described in Sec. VII A, could lead to an important sensitivity improvement and the possibility mentioned in Sec. VI of displacing both beams simultaneously to better evaluate a systematic error may be implemented in the future. This work was supported by Brookhaven Science Associates, LLC, under Contract No. DE-AC02-98CH10886 with the U.S. Department of Energy.
[1] V. Shestak et al., An electron beam probe for ion beam diagnosis, TRIUMF Design Note No. TRI-DN-87-36 Rev, 1987.

[2] J. A. Pasour and M. T. Ngo, Nonperturbing electron beam probe to diagnose charged-particle beams, Rev. Sci. Instrum. 63, 3027 (1992).

[3] E. Tsyganov et al., Electron beam emittance monitor for the SSC, in Proceedings of the 15th Particle Accelerator Conference, PAC-1993, Washington, DC, 1993 (IEEE, New York, 1993), p. 2489.

[4] R. E. Pollock, M. Klasen, J. Lash, and T. Sloan, An Electron probe as a stored beam diagnostic, Nucl. Instrum. Methods Phys. Res., Sect. A 330, 27 (1993).

[5] P. V. Logatchov, P. A. Bak, A. A. Starostenko, N. S. Dikansky, V. S. Tupikov, K. V. Gubin, V. M. Mishnev, M. B. Korabelnikov, and M. G. Fedotov, Non-destructive single pass monitor of longitudinal charge distribution in an ultrsrelativistic electron bunch, in Proceedings of the 18th Particle Accelerator Conference, New York, 1999 (IEEE, New York, 1999), p. 2167.

[6] W. Blokland, A. Aleksandrov, S. Cousineau, D. Malyutin, and S. Starostenko, Electron scanner for SNS ring profile measurements, in Proceedings of the 9th European Workshop on Beam Diagnostics and Instrumentation for Particle Accelerators, Basel, Switzerland, 2009 (PSI, Basel, 2009), TUOA03, https://accelconf.web.cern.ch/accelconf/ d09/papers/tuoa03.pdf.

[7] W. Blokland and S. Cousineau, A non-destructive profile monitor for high intensity beams, in Proceedings of the 24th Particle Accelerator Conference, PAC-2011, New York, 2011 (IEEE, New York, 2011), WEOCN2, http://accelconf .web.cern.ch/accelconf/pac2011/papers/weocn2.pdf.

[8] V. Dudnikov and A. Aleksandrov, Ribbon electron beam profile monitor for bunched beam tomography, in Proceedings of the 3rd International Particle Accelerator Conference, New Orleans, LA, 2012 (IEEE, Piscataway, NJ, 2012), MOPPD048, http://accelconf.web.cern.ch/ accelconf/ipac2012/papers/moppd048.pdf.

[9] E. Rutherford, LXXIX. The scattering of $\alpha$ and $\beta$ particles by matter and the structure of the atom, Philos. Mag. 21, 669 (1911).

[10] P. Thieberger, F. Z. Altinbas, C. Carlson, C. Chasman, M. Costanzo, C. Degen, A. Drees, W. Fischer, D. Gassner, X. Gu, K. Hamdi, J. Hock, Y. Luo, A. Marusic, T. Miller, M. Minty, C. Montag, A. Pikin, and S. White, The electron backscattering detector (eBSD), a new tool for the precise mutual alignment of the electron and ion beams in electron lenses, in Proceedings of IBIC2014, Monterey, $C A$, MOPD02, https://jacowfs.jlab.org/conf/proceedings/ IBIC2014/papers/mopd02.pdf.

[11] W. Fischer, X. Gu, Z. Altinbas, M. Costanzo, J. Hock, C. Liu, Y. Luo, A. Marusic, R. Michno, T. A. Miller, A. I. Pikin, V. Schoefer, and P. Thieberger, Operational Head-on Beam-Beam Compensation with Electron Lenses in the Relativistic Heavy Ion Collider, Phys. Rev. Lett. 115, 264801 (2015).

[12] W. Fischer, X. Gu, S. M. White, Z. Altinbas, D. Bruno, M. Costanzo, J. Hock, A. Jain, Y. Luo, C. Mi, R. Michnoff, T. A. Miller, A. I. Pikin, T. Samms, Y. Tan, R. Than, and $\mathrm{P}$. Thieberger, First experience with electron lenses for beam-beam compensation in RHIC, IPAC2014 
(JACoW, Geneva, 2014), pp. 4046-4048; X. Gu et al., Report No. BNL-10090-2013-CP.

[13] G. Stancari, A. Valishev, G. Annala, G. Kuznetsov, V. Shitsev, D. A. Still, and L. G. Vorobiev, Collimation with Hollow Electron Beams, Phys. Rev. Lett. 107, 084802 (2011); G. Stancari, V. Previtali, A. Valishev, R. Bruce, S. Redaelli, A. Rossi, and B. Salvachua Ferrando, Reports No. CERN-ACC-2014-0248 and No. FERMILAB-TM2572-APC; G. Stancari, Applications of electron lenses: scraping of high-power beams, beam-beam compensation, and nonlinear optics, arXiv:1409.3615.

[14] F. Halzen and A. Martin, Quarks and Leptons (John Wiley and Sons, New York, 1984).

[15] E. Tsyganov, A. Taratin, and A. Zinchenko, SSCL Report No. 519, 1993.

[16] V. Shiltsev, V. Danilov, D. Finley, and A. Sery, Considerations on compensation of beam-beam effects in the Tevatron with electron beams, Phys. Rev. ST Accel. Beams 2, 071001 (1999).

[17] X.-L. Zhang, K. Bishofberger, V. Kamerdzhiev, V. Lebedev, V. Shiltsev, R. Thurman-Keup, and A. Tollestrup, Generation and diagnostics of uncaptured beam in the Fermilab Tevatron and its control by electron lenses, Phys. Rev. ST Accel. Beams 11, 051002 (2008).

[18] W. Fischer et al., CERN Report No. CERN-2014-004, pp. 109-120, 2014.

[19] E. Tsyganov, R. Meinke, W. Nexsen, and A. Zinchenko, SSCL Report No. 519, 1993.

[20] V. Shiltsev, V. Danilov, D. Finley, and A. Sery, Considerations on compensation of beam-beam effects in the Tevatron with electron beams, Phys. Rev. ST Accel. Beams 2, 071001 (1999).

[21] Y. Luo, W. Fischer, N. P. Abreu, X. Gu, A. Pikin, and G. Robert-Demolaize, Six-dimensional weak-strong simulation of head-on beam-beam compensation in the relativistic heavy ion collider, Phys. Rev. ST Accel. Beams 15, 051004 (2012).

[22] X.-L. Zhang et al., Experimental studies of beam-beam effects in the Tevatron, in Proceedings of the Particle Accelerator Conference, Portland, OR, 2003 (IEEE, New York, 2003), p. 1781.

[23] Vladimir Shiltsev, Kip Bishofberger, Vsevolod Kamerdzhiev, Sergei Kozub, Matthew Kufer, Gennady Kuznetsov, Alexander Martinez, Marvin Olson, Howard Pfeffer, Greg Saewert, Vic Scarpine, Andrey Seryi, Nikolai Solyak, Veniamin Sytnik, Mikhail Tiunov, Leonid Tkachenko, David Wildman, Daniel Wolff, and Xiao-Long Zhang, Tevatron electron lenses: Design and operation, Phys. Rev. ST Accel. Beams 11, 103501 (2008).

[24] V. Shiltsev and A. Zinchenko, Electron beam distortions in beam-beam compensation setup, Phys. Rev. ST Accel. Beams 1, 064001 (1998).

[25] J. D. Jackson, Classical Electrodynamics, 3rd ed. (John Wiley \& Sons, Inc., New York, 1999), p. 590, Eq. 12.58.

[26] Hamamatsu PMT, http://www.hamamatsu.com/us/en/ R3998-02.html.

[27] ESTAR data base-NIST Physical Measurement Laboratory (PML), http://physics.nist.gov/PhysRefData/Star/Text/ ESTAR.html.

[28] A. Drees and T. Ottavio, Luminosity optimization using automated IR steering at RHIC, in Proceedings of the 9th European Particle Accelerator Conference, Lucerne, 2004 (EPS-AG, Lucerne, 2004), p. 911.

[29] C. Adler, A. Denisov, E. Garcia, M. Murray, H. Strobele, and S. White, The RHIC zero-degree calorimeters, Nucl. Instrum. Methods Phys. Res., Sect. A 499, 433 (2003).

[30] Ortec Maestro software: http://www.ortec-online.com/ Products-Solutions/Applications-Software-MAESTRO .aspx.

[31] Berkeley Nucleonics Corp. BNC, Model 6010 light pulse generator.

[32] Agilent 53230 A, Universal Frequency Counter/Timer, Agilent Technologies.

[33] G. Stancari, A. Valishev, G. Annala, G. Kuznetsov, V. Shiltsev, D. A. Still, and L. G. Vorobiev, Collimation with hollow electron beams, Phys. Rev. Lett. 107, 084802 (2011).

[34] G. Stancari, in Proceedings of the Meeting of the Division of Particles and Fields of the American Physical Society, Providence, RI, 2011, edited by T. Speer, SLAC eConf C110809 (FNAL, Batavia, IL, 2011); New methods of particle collimation in colliders, arXiv:1110.0144; Report No. FERMILAB-CONF-11-506-AD-APC, 2011.

[35] G. Stancari et al., Electron lenses for the large hadron collider, in Proceedings of the 2014 International Particle Accelerator Conference (IPAC14), Dresden, Germany, 2014, edited by C. Petit-Jean-Genaz, G. Arduini, P. Michel, and V. R. W. Schaa (CERN, Geneva, 2014), p. 918; Report No. FERMILAB-CONF-14-179-APC, 2014.

[36] V. Shiltsev, Electron Lenses for particle collimation in LHC, in Proceedings of the CARE-HHH-APD Workshop (BEAM07), Geneva, Switzerland (CERN, Geneva, 2008), p. 46.

[37] P. Thieberger, C. Chasman, W. Fischer, D. Gassner, X. Gu, M. Minty, and A. Pikin, Scattered electrons as possible probes for beam halo diagnostics, in Workshop on Beam Halo Monitoring (SLAC National Accelerator Laboratory, Menlo Park, CA, 2014).

[38] S. Turner, CERN Accelerator School Vacuum Technology, Scatnticon Conference Centre, Snekkersten, Denmark, 1999, http://www.iaea.org/inis/collection/NCLCollectionStore/_ Public/32/011/32011645.pdf.

[39] J. P. Koutchouck, Correction of the long-range beam-beam effect in LHC using electromagnetic lenses, in Proceedings of the 19th Particle Accelerator Conference, Chicago, IL, 2001 (IEEE, Piscataway, NJ, 2001), p. 1683.

[40] W. H. DeLuca, Beam detection using residual gas ionization, IEEE Trans. Nucl. Sci. 16, 813 (1969).

[41] D. D. Chamberlin G. N. Minerbo, L. E. Teel, and J. D. Gilpatrick, Noninterceptive transverse beam diagnostics, IEEE Trans. Nucl. Sci. 28, 2347 (1981).

[42] M. Ferro-Luzzi, Proposal for an absolute luminosity determination in colliding beam experiments using vertex detection of beam-gas interactions, Nucl. Instrum. Methods Phys. Res., Sect. A 553, 388 (2005).

[43] LHCb Collaboration, Precision luminosity measurements at LHCb, J. Instrum. 9, P12005 (2014).

[44] P. Hopchev et al., A beam gas vertex detector for beam size measurement in the LHC, in Proceedings of IPAC2014, Dresden, Germany, THPME175.

[45] E. C. Aschenauer et al., eRHIC design study: An ElectronIon Collider at BNL, arXiv:1409.1633. 